\title{
Diet advice in colorectal cancer survivors - are we doing enough?
}

\author{
S.L. Matsel1 ${ }^{1,2}$, M.A. Sanchez Garcia ${ }^{1,2}$, Dr E.A. Williams ${ }^{1}$ and Dr B.M. Corfe ${ }^{2}$ \\ ${ }^{1}$ Human Nutrition Unit, Department of Oncology and Metabolism, University of Sheffield, The Medical School, \\ Beech Hill Road Sheffield, S10 2RX, UK and \\ ${ }^{2}$ Molecular Gastroenterology Research Group, Department of Oncology, University of Sheffield, The Medical School, \\ Beech Hill Road Sheffield, S10 2RX, UK.
}

Research on nutrition and cancer survival is at an early stage ${ }^{(1)}$. Current dietary recommendations for colorectal cancer focus on primary prevention, leaving a gap in the advice to be given to survivors. The NIHR Infrastructure Collaboration found $72 \%$ of allcancer patients in the UK did not receive nutritional advice or support ${ }^{(2)}$. Considering the gastrointestinal and nutritional implications associated with colorectal cancer therapy, the present study aimed to evaluate the quantity and quality of advice being received by UK colorectal cancer patients and survivors.

A modified version of a previous survey ${ }^{(2)}$ was used, and was available online and in paper copies between May and August 2017. The survey contained open-ended and closed-ended questions, which explored the nutritional problems that the participants had experienced, as well as their opinion regarding the advice that was available for colorectal cancer patients. Recruitment was conducted by contacting support groups, including visits, and through cancer charities on social media.

Seventy-nine colorectal cancer patients completed the survey. Seventy percent of them had not received nutritional support from their healthcare team. Those who had received advice expressed mixed opinions regarding the quality. Quantitative and qualitative data testified substantial differences in the nutritional problems experienced by colorectal cancer patients, such as diarrhoea, constipation and stoma related difficulties. These would often hinder the ability of following healthy diet guidelines. Food intolerances and other bowel impairments changed between patients and throughout diagnosis, treatment and post-treatment, demanding specificity and adaptability of the advice. General information available online or provided in leaflets would not meet their individual requirements. Participants reported lack of information, and need for individualised advice available at each stage and long-term post-treatment.

The digestive difficulties experienced by colorectal cancer patients requires specialised advice which is currently not being provided. Tailored dietary interventions have shown to be feasible ${ }^{(3)}$, and are demanded by colorectal cancer survivors. There is a need for tailored advice which facilitates a healthier lifestyle and follows up their needs from diagnosis.

1. World Cancer Research Fund / American Institute for Cancer Research. Washington DC: AICR; 2007.

2. Cancer and Nutrition NIHR infrastructure collaboration. Report of Phase One July. 2015.

3. Vijayvergia N., Denlinger C.S. (2015). J. Pers. Med. 5, 243-63. 